\title{
PASSIVE LOCALIZATION FOR MIXED-FIELD MOVING SOURCES
}

\author{
Chunxian Gao, Ph.D, \\ Hui Liu, Ph.D, \\ Communication Engineering Department, Xiamen University, Xiamen, Fujian, China
}

\begin{abstract}
Due to the mobility of underwater equipment, high-precision underwater positioning technology will face two technical challenges: dealing with mixed-field signals composed of near-field signals and far-field signals; adapting to variable component of mixed-field signals considering the mobility of equipment. Under this condition, an effective method based on MUSIC is addressed in this paper. After distinguishing far-field signal subspace from mixed-field signal subspace, estimations of DOAs and powers of far-field sources are carried out. Then the corresponding far-field and noise signal components can be eliminated from the signal subspace. After that, based on path-following algorithm, modified 2D-MUSIC is performed for DOA and range estimations of near-field sources. The performance of the proposed method is verified and compared with the other methods through computer simulations. Reasonable classification of source types and accurate localization estimation can be achieved by using the proposed method.
\end{abstract}

Keywords: underwater positioning; passive localization; mixed-field sources; MUSIC algorithm

\section{INTRODUCTION}

Underwater smart equipment has technical advantages in marine environmental monitoring, seabed mineral exploration, and harbor monitoring, which mostly includes UUVs, ROVs and AUVs. With the advancement of underwater communication technology and battery technology, more and more underwater equipment has been deployed to offshore ports and open ocean areas, which has driven the rapid development of the marine industry. With the release of a large number of equipment, high-precision underwater positioning technology has become the core technology for underwater equipment monitoring, joint operation and equipment autonomous operation.
Source localization based on array signal is a fundamental problem in many fields such as radar, sonar, communications, and seismic exploration [1]. In the past decades, a great deal of work has been carried out in this field and various algorithms have been proposed for either far-field sources [1]-[3] or near-field sources [4]-[8]. However, in most practical applications, sources may be spread over a wide area, creating a mixed-filed signal environment. In some special cases, some of the sources may switch between far-field and near-field range continuously due to random movement of sources in that area. Most methods aforementioned may fail or deteriorate in such environment.

Recently, passive localization under mixed-field sources environment has been explored, and several efficient methods 
are available. A two-stage MUSIC method [9] was proposed by Liang and Liu, to solve the mixed-field sources localization problem. Wang and Sun [10] reconstructed and utilized the sparsity of mixed-field signal subspace, and the better performance of accuracy and resolution were proved. He and Swanmy [11] introduced second-order statistics (SOS) to their solution and improved computation efficient. By implementing matrix difference technology, two-stage localization algorithm was proposed by Liu and Sun [12]. However, these methods are dependent on the hypothesis that the exact numbers of far-field sources or near-field sources are known in advance and keep invariant. In reality the exact numbers remain in a state of flux because of the possible motion of sources. Thus, these methods would encounter the failure problem or unsatisfactory performance.

In this paper, a new method was proposed for the mixedfield sources environment where some of them may be in motion. By exploring the difference of MUSIC spectral features between far-field and near-field signals, an estimator, termed as far-field distinguisher, is constructed to distinguish far-field signals from mixed-field signals. Azimuth DOAs and powers of far-field sources are estimated and eliminated from the mixed signal subspace. Based on path-following algorithm, azimuth DOAs and ranges information of nearfield sources are obtained via Strip-2D-MUSIC searching. Computer simulation proves that the proposed method avoids the estimation failure problem and provides a more accurate estimation performance.

The remainder of the paper is organized as follows. Section II describes the signal model for localization estimation of mixed-field sources. Section III derives the algorithms involved in the proposed method and introduces its main implementation steps. Section IV shows the computer simulation results. Section $\mathrm{V}$ is the conclusion of the whole paper.

\section{SIGNAL MODELS}

There are $\mathrm{M}$ uncorrelated narrowband sources under mixed-field sources scenarios, among which there are $M_{N}$ near-field signals and $\left(M-M_{N}\right)$ far-field signals. The sensor array is a symmetric uniform linear array and consists of $\mathrm{L}=2 \mathrm{~N}+1$ sensors with inter-sensor spacing $\mathrm{d}$. The phase reference point of the sensor array is set in its center. The sample signal received by sensor array can be written as $[11,12]$

$$
\mathbf{x}(t)=\mathbf{A}_{N} \mathbf{s}_{N}(t)+\mathbf{A}_{F} \mathbf{s}_{F}(t)+\mathbf{n}(t)
$$

where

$$
\begin{gathered}
N \quad\left[s(t) \cdots s_{m}(t) \cdots s_{M}(t)\right] \\
\mathbf{s}_{F}=\left[s_{M_{N}+1}(t) \cdots s_{M_{N}+m}(t) \cdots s_{M}(t)\right]^{T}
\end{gathered}
$$

$$
\mathbf{A}_{N}=\left[\mathbf{a}\left(\theta_{1}, r_{1}\right) \cdots \mathbf{a}\left(\theta_{m}, r_{m}\right) \cdots \mathbf{a}\left(\theta_{M_{N}}, r_{M_{N}}\right)\right]
$$

$$
\mathbf{A}_{F}=\left[\mathbf{a}\left(\theta_{M_{N}+1}\right) \cdots \mathbf{a}\left(\theta_{M_{N}+m}\right) \cdots \mathbf{a}\left(\theta_{M}\right)\right]
$$

$\mathbf{S}_{N}$ and $\mathbf{S}_{F}$ are near-field and far-field source signal vectors, respectively. $\mathbf{A}_{N}$ and $\mathbf{A}_{F}$ are the array steering matrices of near-field and far-field sources, respectively. $\mathbf{a}\left(\theta_{m}, r_{m}\right)$ and $\mathbf{a}\left(\theta_{m}\right)$ are the array steering vectors for near-field and far-field sources, respectively.

$$
\begin{gathered}
\mathbf{a}\left(\theta_{m}, r_{m}\right)=\left[e^{j\left(-N \gamma_{m}+N^{2} \phi_{m}\right)}, \cdots, 1, \cdots, e^{j\left(N \gamma_{m}+N^{2} \phi_{m}\right)}\right]^{T} \\
\mathbf{a}\left(\theta_{m}\right)=\left[e^{-j N \gamma_{m}}, \cdots, 1, \cdots, e^{j N \gamma_{m}}\right]^{T} \\
\gamma_{m}=-2 \pi(d / \lambda) \sin \theta_{m} \\
\phi_{m}=\pi\left[d^{2} /\left(\lambda r_{m}\right)\right] \cos ^{2} \theta_{m}
\end{gathered}
$$

where $\lambda$ is the wavelength of the source signal, $\theta_{m}$ and $r_{m}$ stand for DOA and range of the mth signal source. $\mathbf{n}(t)$ is additive sensor noise and modeled as zero-mean Gaussian which is spatially uniformly white and statistically independent of all the signals.

Based on (1), the array covariance matrix can be calculated as

$$
\begin{aligned}
\mathbf{R} & =E\left\{\mathbf{x}(t) \mathbf{x}^{H}(t)\right\} \\
& =\mathbf{A}_{N} \mathbf{S}_{N} \mathbf{A}_{N}^{H}+\mathbf{A}_{F} \mathbf{S}_{F} \mathbf{A}_{F}^{H}+\sigma_{n}^{2} \mathbf{I} \\
& =\mathbf{R}_{N}+\mathbf{R}_{F}+\sigma_{n}^{2} \mathbf{I} \\
& =\mathbf{A} \mathbf{S} \mathbf{A}^{H}+\sigma_{n}^{2} \mathbf{I}
\end{aligned}
$$

Where $\mathbf{S}, \mathbf{S}_{N}$ and $\mathbf{S}_{F}$ are diagonal matrices and diagonal elements represent power of corresponding sources. $\sigma_{n}^{2}$ indicates the sensor noise power.

\section{PROPOSED SOLUTION}

\section{NOISE POWER ESTIMATION}

The proposed solution assumes that the value of $M$ is known, but the actual number of far-field or near-field sources remains unknown because of the possible motion of sources. The array covariance matrix can be eigen decomposed as 


$$
\mathbf{R}=\mathbf{U}_{S} \Delta_{s} \mathbf{U}_{S}^{H}+\mathbf{U}_{N} \Delta_{n} \mathbf{U}_{N}^{H}
$$

where $\Delta_{s} \in R^{M \times M}$ is a diagonal matrix and its diagonal elements are the $M$ largest eigenvalues of $\mathbf{R}$, $\Delta_{n} \in R^{(L-M) \times(\mathrm{L}-M)}$ is a diagonal matrix and its diagonal elements are the rest eigenvalues of $\mathbf{R}, \mathbf{U}_{S} \in C^{L \times M}$ spans the signal subspace which consists of the eigenvectors related to the $\mathrm{M}$ largest eigenvalues, and $\mathbf{U}_{n} \in C^{\mathrm{L \times (L-M)}}$ spans the noise subspace which consists of the eigenvectors related to the rest. Based on the MUSIC subspace theory [2], power of noise can be calculated as

$$
\tilde{\sigma}_{n}^{2}=\frac{1}{L-M} \operatorname{tr}\left(\Delta_{n}\right)
$$

Then $\tilde{\mathbf{R}}_{F N}$ can be reconstructed which only contains the far-field and near-field signal information.

$$
\tilde{\mathbf{R}}_{F N}=\mathbf{U}_{S}\left(\Delta_{s}-\tilde{\sigma}_{n}^{2} \mathbf{I}_{\mathrm{M} \times \mathrm{M}}\right) \mathbf{U}_{S}^{H}
$$

It can be seen from (13) that sensor noise is well eliminated, and its influence is effectively reduced.

\section{DOA ESTIMATION FOR FAR-FIELD SOURCES}

According to 2D-MUSIC pseudo-spectrum scalar function [4], far-field source signal will exhibit peaks at the range $r=\infty$ with corresponding azimuth. 2D-MUSIC pseudospectrum scalar function defined as

$$
V(\theta, r)=\left[\mathbf{a}^{H}(\theta, r) \tilde{\mathbf{U}}_{F N n} \tilde{\mathbf{U}}_{F N n}^{H} \mathbf{a}(\theta, r)\right]^{-1}
$$

where $\tilde{\mathbf{U}}_{F N n}$ represents the noise eigenvector matrix of $\tilde{\mathbf{R}}_{F N}$. This indicates that the DOAs of the far-field sources can be obtained by screening all peaks from the following 1D-MUSIC pseudo-spectrum function,

$$
P(\theta)=\left[\mathbf{a}^{H}(\theta, \infty) \tilde{\mathbf{U}}_{F N n} \tilde{\mathbf{U}}_{F N n}^{H} \mathbf{a}(\theta, \infty)\right]^{-1}
$$

In our scenarios, there are two fatal flaws in (15). Firstly, there exists spurious peaks among real peaks due to the interference from near-field signals or noise, which is deduced and simulated in paper [13]. The phenomenon of spurious peaks would lead to estimation failure. Secondly, the number of far-field sources remains unknown during estimating. Sorting out the first k maximum peaks or using conventional methods, which would not solve far-field estimation problem. For confirming estimations from (15), further processing need to be carried out. For each azimuth $\theta_{i}$ and corresponding pseudo-spectrum value $V\left(\theta_{i}, \infty\right)$ from (15), we do another $1 \mathrm{D}$ searching on range dimension, termed as far-field signal distinguisher,
$V\left(\theta_{i}, r_{i}\right)=\max _{r}\left\{\left[\mathbf{a}^{H}\left(\theta_{i}, r\right) \tilde{\mathbf{U}}_{F N n} \tilde{\mathbf{U}}_{F N n}^{H} \mathbf{a}\left(\theta_{i}, r\right)\right]^{-1}\right\}$

where $r \in\left[0.62\left(D^{2} / \lambda\right)^{1 / 2}, 2 D^{2} / \lambda\right]$ and $D$ represents the array aperture. Finally, far-field sources classification and DOA estimation are fulfilled based on the following criteria,

$$
\left\{\begin{array}{l}
\tilde{\theta}_{F}=\theta_{i}, \text { far-field, if } V\left(\theta_{i}, \infty\right)>V\left(\theta_{i}, r_{i}\right) \\
\text { spurious peak, if } V\left(\theta_{i}, \infty\right) \leq V\left(\theta_{i}, r_{i}\right)
\end{array}\right.
$$

\section{POWER ESTIMATION FOR FAR-FIELD SOURCES}

After processing of (13), far-field signals become the largest interference sources for the parameters estimation of nearfield sources. Removal of far-field signals from mixed-field signals subspace is helpful to improve the accuracy of nearfield parameters estimation. Similar to the mathematical derivation in paper [13], the power of mth far-field signal can be estimated as,

$$
\begin{aligned}
\tilde{\sigma}_{m}^{2} & =\frac{1}{\tilde{\mathbf{a}}^{H}\left(\tilde{\theta}_{m}\right)\left[\mathbf{A S A} \mathbf{A}^{H}\right]^{+} \tilde{\mathbf{a}}\left(\tilde{\theta}_{m}\right)} \\
& =\frac{1}{\tilde{\mathbf{a}}^{H}\left(\tilde{\theta}_{m}\right)\left[\tilde{\mathbf{R}}_{F N}\right]^{+} \tilde{\mathbf{a}}\left(\tilde{\theta}_{m}\right)}
\end{aligned}
$$

where $\tilde{\sigma}_{m}^{2}$ is mth far-field source power estimation and $\tilde{\mathbf{a}}\left(\tilde{\theta}_{m}\right)$ is the corresponding estimated steering vector, superscript ${ }^{+}$denotes the pseudo inverse operation. After power estimation of all far-field sources, $\tilde{\mathbf{R}}_{N}$ which only contains near-field information can be reconstructed as

$$
\begin{aligned}
\tilde{\mathbf{R}}_{N} & =\tilde{\mathbf{R}}_{F N}-\tilde{\mathbf{R}}_{F} \\
& =\tilde{\mathbf{R}}_{F N}-\tilde{\mathbf{A}}_{F} \operatorname{diag}\left(\tilde{\sigma}_{1}^{2}, \cdots, \tilde{\sigma}_{M-M_{N}}^{2}\right) \tilde{\mathbf{A}}_{F}^{H}
\end{aligned}
$$

\section{DOA AND RANGE ESTIMATION FOR NEAR-FIELD SOURCES}

According to path-following method $[13,14]$, the algebraic relation between the azimuth $\tilde{\theta}_{i}$ from $1 \mathrm{D}$ MUSIC pseudospectrum function and the actual azimuth $\theta_{i}$ and range $r_{i}$ for near-field source satisfies

$$
\sin \theta_{i}=\sin \tilde{\theta}_{i}+0.5 \times\left(1-\sin ^{2} \tilde{\theta}_{i}\right)\left(D / r_{i}\right)
$$

The numerical difference between $\tilde{\theta}_{i}$ and $\theta_{i}$ is small which is proved in paper [14]. Azimuth estimation from 1D MUSIC pseudo-spectrum function can be regard as rough azimuth estimation for near-field source. Based on this assumption, we propose Strip-2D MUSIC for parameters estimation for 
near-field sources. Firstly, $M_{N}$ azimuths are estimated from 1D MUSIC pseudo-spectrum function,

$$
P(\theta)=\left[\mathbf{a}^{H}(\theta) \tilde{\mathbf{U}}_{N n} \tilde{\mathbf{U}}_{N n}^{H} \mathbf{a}(\theta)\right]^{-1}
$$

where $\tilde{\mathbf{U}}_{N n}$ represents the noise eigenvector matrix of $\tilde{\mathbf{R}}_{N}$. Each estimated azimuth $\theta_{i \text { rough }}$ is termed as rough azimuth estimation for near-field sources. Then, 2D search zone based on rough azimuth estimations is set up,

$$
\left\{\begin{array}{l}
r \in\left[0.62\left(D^{2} / \lambda\right)^{1 / 2}, 2 D^{2} / \lambda\right] \\
\theta \in\left[\theta_{i_{-} \text {rough }}-\Delta \theta, \theta_{i_{-} \text {rough }}+\Delta \theta\right]
\end{array}\right.
$$

Finally, 2D MUSIC search on each zone is carried out, and DOA and range estimation for near-field sources are fulfilled.

$$
V\left(\theta_{i}, r_{i}\right)=\max _{\theta, r}\left\{\left[\mathbf{a}^{H}(\theta, r) \tilde{\mathbf{U}}_{N n} \tilde{\mathbf{U}}_{N n}^{H} \mathbf{a}(\theta, r)\right]^{-1}\right\}
$$

Compared with traditional 2D MUSIC, Strip-2D MUSIC avoids whole plane searching, and its computational complexity is far less than the traditional method.

\section{E. DESCRIPTION OF THE PROPOSED METHOD}

We can summarize the proposed method as follows.

Step 1: $\quad$ Compute the array covariance matrix $\mathbf{R}$ by (10). Step 2: Eigen decompose $\mathbf{R}$, and obtain the power estimation of noise by (12), then eliminate noise by (13).

Step 3: Eigen decompose $\tilde{\mathbf{R}}_{F N}$, and obtain all peaks by (15), then do range dimension search for each estimated azimuth value by (16), finally fulfill far-field signal classification and azimuth DOA estimations by (17).

Step 4: Obtain far-field power estimations by (18), and eliminate far-field signals by (19).

Step 5: Eigen decompose $\widetilde{\mathbf{R}}_{N}$, and obtain $\mathrm{M}_{\mathrm{N}}$ peaks by (21), then set up search zones by (22), finally do Strip-2D MUSIC by (23).

\section{COMPUTER SIMULATION RESULTS}

In this section, the feasibility of the proposed method is verified by computer simulation. A nineteen-element ULA with a quarter-wavelength inter-sensor spacing is used. Before the start of the simulation, there are 5 signal sources to be located. Among them there are three far-field sources and two near-field sources. Three far-field sources are assumed to have the following azimuths: $\left\{-33^{\circ}, 22^{\circ}, 60^{\circ}\right\}$ and two nearfield sources are assumed to be located at $\left(-25^{\circ}, 12 \lambda\right)$ and $\left(13^{\circ}\right.$, $7 \lambda)$. In order to simulate the motion of sources, the position of the source is disturbed before each independent estimation, and the amount of disturbance is assume as $(x \bullet \Delta \theta, x \bullet \Delta \lambda)$ and $x \sim U(-1,1)$.The additive noise is assumed to be spatial white complex Gaussian, and the SNR is defined relative to each signal. For comparison, both the SOS based method (SOS) [11] and the Matrix-Differencing based method (MD) [12] are also presented. The application of SOS or MD depends on the number information of far-filed and near-field sources. At each estimation, SOS and MD is provided with the accurate number of sources, but the proposed method is kept in the state of blind estimation. The presented results are evaluated by the estimated root mean percent square error (RMPSE), defined as

$$
R M P S E=\sqrt{\frac{1}{N} \sum_{i=1}^{N}\left(\frac{E_{i}-R_{i}}{R_{i}}\right)^{2}} \times 100 \%
$$

where denotes the estimated value, denotes the real value and denotes the number of independent estimations.

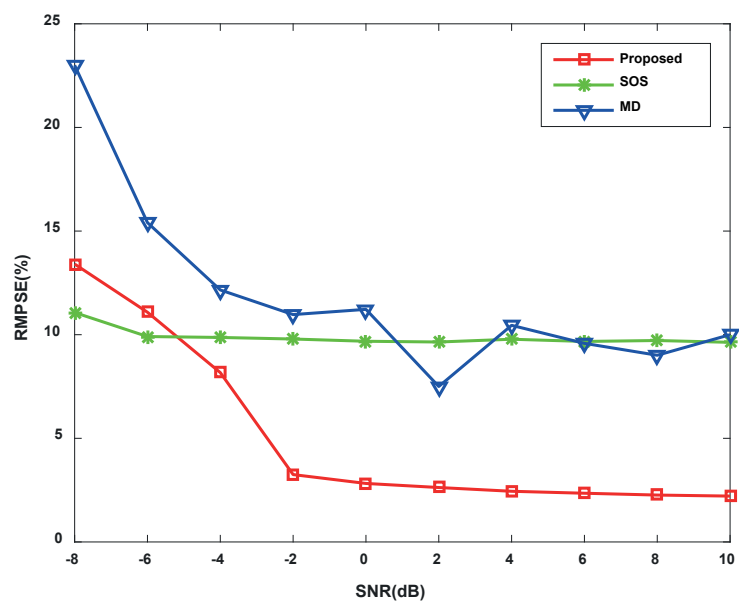

Fig. 1. The RMPSEs of azimuth estimations for far-field sources versus SNRs, the snapshot number is 500, 500 independent trials.

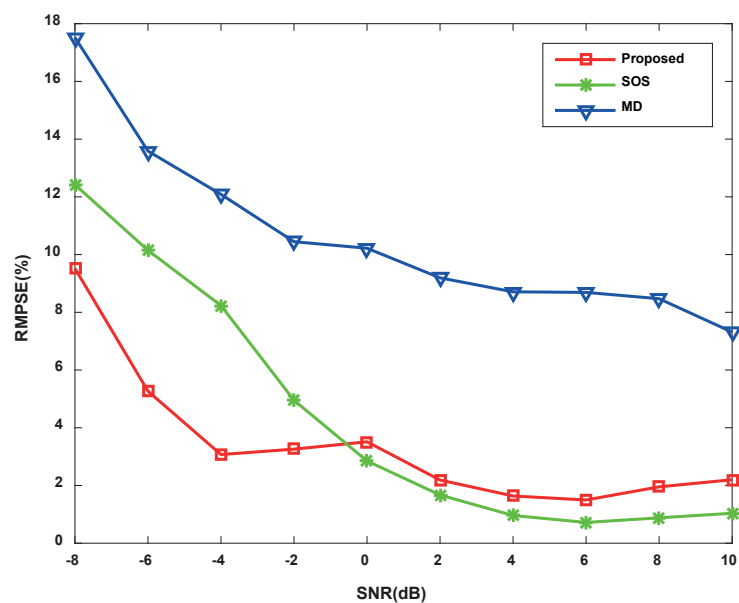

Fig. 2. The RMPSEs of azimuth DOAs estimations for near-field sources versus SNRs, the snapshot number is 500, 500 independent trials. 


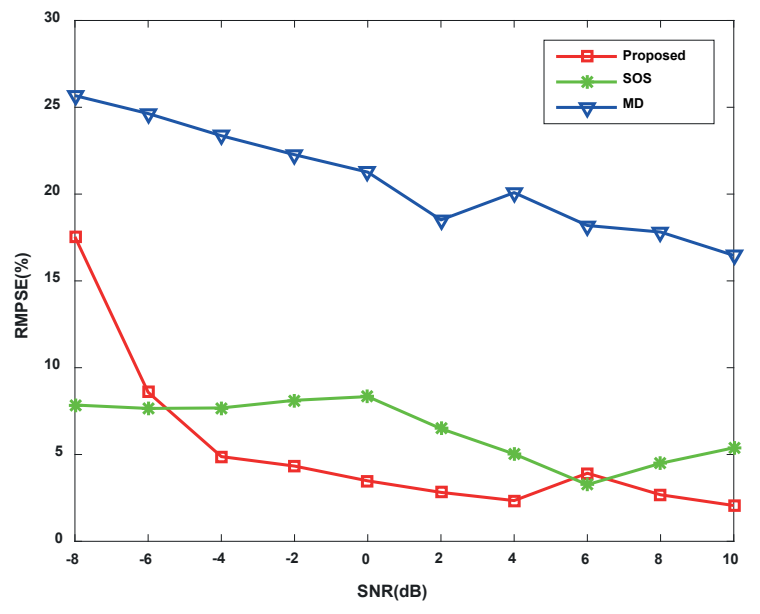

Fig. 3. The RMPSEs of range estimations for near-field sources versus SNRs, the snapshot number is 500, 500 independent trials.

In the first experiment, we examine the performance of three methods under various SNR. Figs. 1-3 respectively show the RMPSE simulation results of far-field sources azimuth estimation, near-field sources azimuth estimation and nearfield sources range estimation under various SNR conditions. From the simulation results, it can be seen that the estimation performance of the three methods has been improved with the increase of SNR. Among them, the performance of the proposed and SOS is better than that of SOS in all kinds of SNR conditions. For far-field azimuth estimation and near-filed range estimation, the performance of the proposed is slightly lower than that of SOS at low SNR condition $(<-6 \mathrm{~dB})$, but with the increase of SNR, the performance of the proposed is better than that of SOS. For near-field azimuth estimation, the performance of the proposed is superior to or equivalent to that of SOS at all SNR conditions. In general, the comprehensive performance of the proposed in all kinds of SNR conditions is better than that of SOS or MD, showing the robustness of the method.

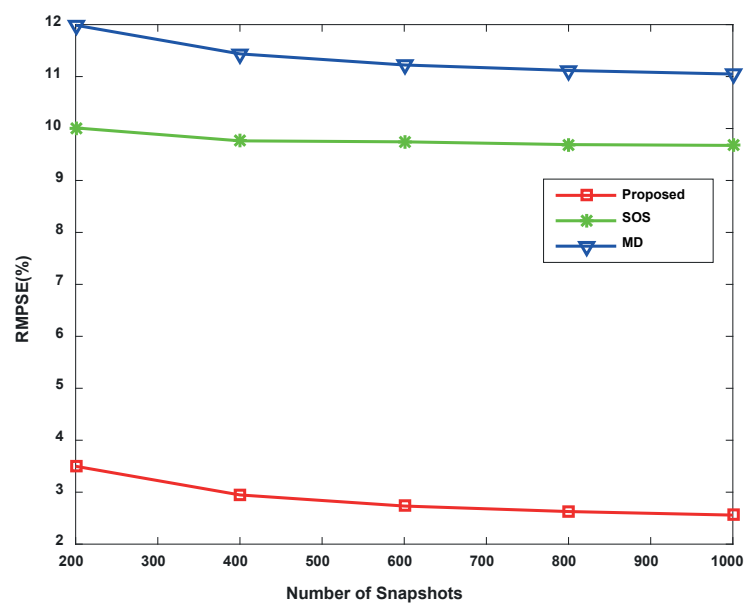

Fig. 4. The RMPSEs of azimuth DOAs estimations for far-field sources versus snapshot number, $S N R=0 d B, 500$ independent trials.

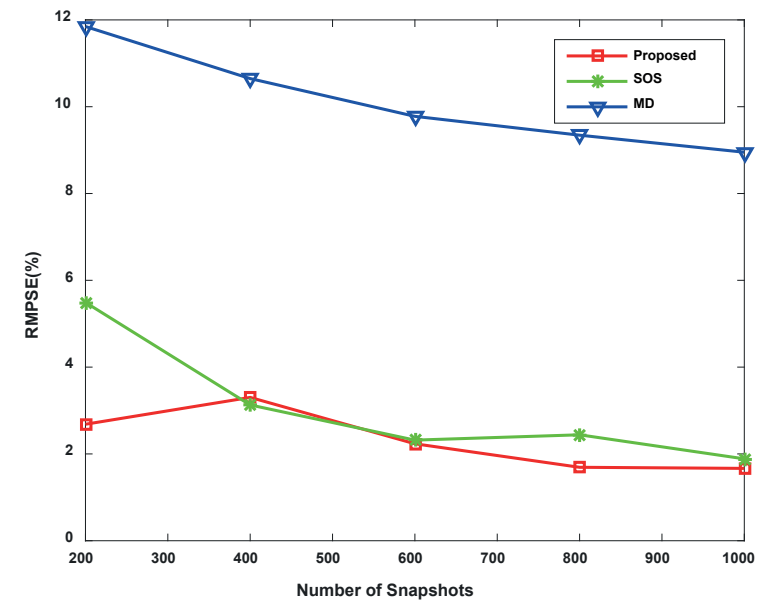

Fig. 5. The RMPSEs of azimuth DOAs estimations for near-field sources versus snapshot number, $S N R=0 d B, 500$ independent trials.

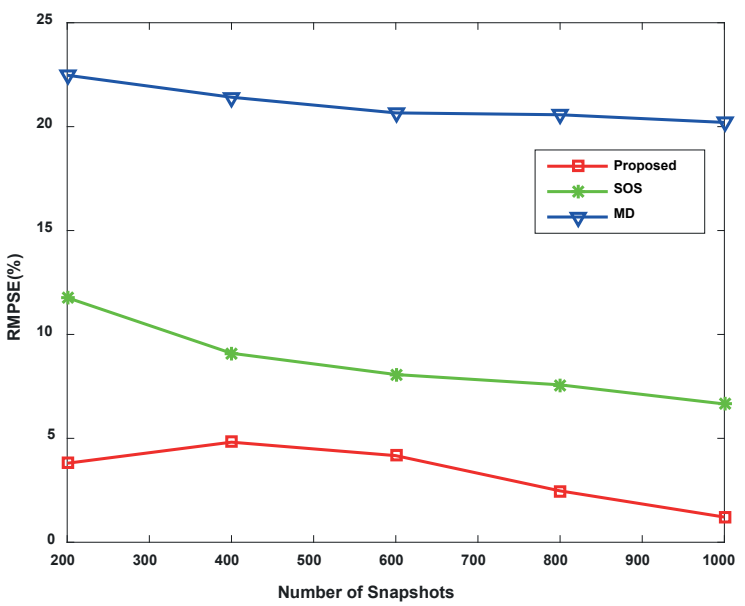

Fig. 6. The RMPSEs of range estimations for near-field sources versus snapshot number, $S N R=0 d B, 500$ independent trials.

Secondly, we assess the performance of the proposed method versus the number of snapshots. The simulation conditions are similar to the first experiment except that the SNR is set at $0 \mathrm{~dB}$, and the number of snapshots is evenly spaced from 200 to 1000 with interval 200. The RMPSE simulation results are respectively displayed in Figs. 4-6. The estimation RMPSE for three methods declines monotonously with the increase of snapshot size. Increasing the size of snapshot does improve the performance of three estimation methods. In the smaller snapshot zone, the performance is improved obviously with the increase of snapshot size. However, when the snapshot size reaches a certain value, the effect of the increase in size tends to be slow.

\section{CONCLUSION}

We have presented a method for mobile sources localization problem under mixed-field scenarios. Our investigation has shown that the proposed method is capable of yielding 
reasonably good estimates of the azimuth and the range estimation for both the far-field and near-field sources.

\section{BIBLIOGRAPHY}

1. H. Krim and M. Viberg, "Two decades of array signal processing research: The parametric approach," IEEE Signal Process. Mag., vol. 13, no. 4, pp. 67-94, Jul. 1996.

2. R. O. Schmidt, "Multiple emitter location and signal parameter estimation," IEEE Trans. Antennas Propag., vol. AP-34, pp. 276-280, Mar. 1986.

3. R. Roy and T. Kailath, "ESPRIT-estimation of signal parameters via rotational invariance techniques," IEEE Trans. Acoust., Speech, Signal Process., vol. 37, pp. 984-995, Jul. 1989.

4. Y. D. Huang and M. Barkat, "Near-field multiple sources localization by passive sensor array," IEEE Trans. Antennas Propag., vol. 39, pp. 968-975, Jul. 1991.

5. A. J. Weiss and B. Friedlander, "Range and bearing estimation using polynomial rooting," IEEE J. Ocean. Eng., vol. 18, no. 2, pp. 130-137, Apr. 1993.

6. E. Grosicki, K. Abed-Meraim, and Y. Hua, "A weighted linear prediction method for near-field source localization," IEEE Trans. Signal Process., vol. 53, no. 10, pp. 3651-3660, Oct. 2005.

7. W. Zhi and M. Y. W. Chia, "Near-field source localization via symmetric subarrays," IEEE Signal Process. Lett., vol. 14, no. 6, pp. 409-412, Jun. 2007.

8. Y. Wu, H. C. So, and J. Li, "Passive localization of near-field sources with a polarization sensitive array," IEEE Trans. Antenna Propag., vol. 55, no. 8, pp. 2402-2408, Aug. 2007.

9. J. Liang and D. Liu, "Passive localization of mixed near-field and far-field sources using two-stage music algorithm," IEEE Trans. Signal Process., vol. 58, no. 1, pp. 108-120, Jan. 2010.

10. B. Wang, J. Liu, and X. Sun, "Mixed sources localization based on sparse signal reconstruction," IEEE Signal Process. Lett., vol. 19, no. 8, pp. 487-490, Aug. 2012.

11. J. He, M. N. S. Swany, and M. O. Ahmad, "Efficient application of MUSIC algorithm under the coexistence of far-field and near-field sources," IEEE Trans. Signal Process., vol. 60, no. 4, pp. 2066-2070, Apr. 2012.

12. G. Liu and X. Sun, "Two-Stage Matrix Differencing Algorithm for Mixed Far-Field and Near-Field Sources Classification and Localization," IEEE Sensors J., vol. 14, no. 6, pp. 1957-1965, Jun. 2014.
13. D. Starer and A. Nehorai, "Passive Localization of NearField Sources by Path Following," IEEE Trans. Signal Process., vol. 42, no. 3, Mar. 1994.

14. J. H. Lee, C.-M. Lee, and K.-K. Lee, "A path-following algorithm using a known algebraic path," IEEE Trans. Acoust., Speech Signal Process., vol. 47, no. 5, pp. 14871490, May 1999.

\section{CONTACT WITH THE AUTHORS}

Hui Liu, Ph.D

email:lh@xmu.edu.cn tel.: +86-13860494875

Communication Engineering Department Xiamen University Xiamen Fujian, 361005

\section{China}

